\title{
RECOVERING REFINED SURFACE NORMALS FOR RE- LIGHTING CLOTHING IN DYNAMIC SCENES
}

\author{
P. Csakany ${ }^{1}$, F. Vajda ${ }^{2}$ and A. Hilton ${ }^{1}$ \\ ${ }^{1}$ University of Surrey, Centre for Vision speech and Signal Processing, Guildford, UK \\ ${ }^{2}$ Budapest University of Technology and Economics, Department of Control Engineering and Information Technology, \\ Budapest, Hungary
}

Keywords: Relighting, normal map recovery, shape-fromshading, view consistent segmentation

\begin{abstract}
In this paper we present a method to relight captured 3D video sequences of non-rigid, dynamic scenes, such as clothing of real actors, reconstructed from multiple view video. A viewdependent approach is introduced to refine an initial coarse surface reconstruction using shape-from-shading to estimate detailed surface normals. The prior surface approximation is used to constrain the simultaneous estimation of surface normals and scene illumination, under the assumption of Lambertian surface reflectance. This approach enables detailed surface normals of a moving non-rigid object to be estimated from a single image frame. Refined normal estimates from multiple views are integrated into a single surface normal map. This approach allows highly non-rigid surfaces, such as creases in clothing, to be relit whilst preserving the detailed dynamics observed in video.
\end{abstract}

\section{Introduction}

Entertainment content production requires the compositing of real and computer generated elements under the same illumination conditions to achieve realistic rendering. Over the past decade there has been extensive research in computer vision and graphics on reconstruction of moving people from multiple view video [1][2][3][4][5]. These approaches allow performance capture and replay from novel viewpoints. Commonly the scene is rendered with the captured appearance under the fixed illumination of the captured multi-view video using view-dependent texture mapping. This prohibits realistic compositing in scenes with different illumination conditions. In this paper we introduce a novel video-based approach for relighting multiple view reconstructions of people. Our aim is to enable captured representations of real actors to be seamlessly composited in real or computer generated scenes.

Existing methodologies for reconstruction of surface reflectance properties use techniques such as photo-metric stereo [6] which requires the scene to be captured in full correspondence under multiple illumination conditions. This limits the approach to static scenes where multiple images can be captured for different light sources. State-of-the-art multiple view performance capture and reconstruction methodologies [5][6][7] produce models of sufficient accuracy for realistic rendering but do not reconstruct surface normals with sufficient accuracy for relighting of dynamic scenes. Recent research has addressed the problem of reflectance estimation for non-rigid moving scenes by either integrating multiple view temporal observations of surface points [8][9] or using specialist highspeed cameras and illumination [10]. Integration of multiple view observations over time requires accurate shape reconstruction and temporal surface correspondence both unsolved problems for surfaces with uniform appearance resulting in loss of surface detail. The use of specialist highspeed camera and illumination rigs limits the capture volume and is prohibitively expensive.

In this paper we propose a video-based approach to relighting non-rigid scenes which utilises standard $25 \mathrm{~Hz}$ video capture and studio illumination. The approach combines an initial multiple view shape reconstruction [6] with shape-fromshading to estimate the detailed surface normals for relighting. The novelty of this approach is the use of a prior shape model to constrain both normal and scene illumination estimation. This allows the reconstruction of detailed surface normals from a single image without requirements for accurate spatial or temporal correspondence, under the assumption of Lambertian reflectance. Multiple view reconstruction of detailed surfaces normals are integrated into a single consistent surface normal map at each time frame. The resulting representation can then be rendered under novel illumination conditions. The videobased reconstruction of surface normals at each image frame in a video sequence enables relighting of surface detail such as dynamic creases in clothing which was not possible with previous techniques. Contributions of this approach include a novel model based normal-from-shading estimation for Lambertian surfaces, single time-frame estimation of detailed surface under arbitrary illumination conditions and multiple view integration into a single surface normal map for relighting.

\section{Previous Work}

Over the past decade there has been extensive research in reconstruction of moving people captured in multiple camera studios [1][2][3][4][5][7][8]. These approaches reconstruct sequences of textured 3D surfaces using stereo and silhouette based reconstruction techniques enabling free-viewpoint visualisation or Virtualized Reality ${ }^{\mathrm{TM}}$ [1]. Current methodologies for reconstruction of surface sequences do not recover non-rigid surface detail such as creases in clothing with sufficient accuracy to allow reconstruction of surface reflectance properties. This is due to the inherent ambiguity of stereo correspondence for uniform surface regions.

Reconstruction of surface reflectance requires accurate knowledge of both the surface shape (normals) and the scene illumination, which has only previously been demonstrated for static scenes [11]. The dynamic appearance of surface detail is 
commonly represented as texture map images corresponding to the observed surface irradiance which depends on both the intrinsic surface reflectance and illumination conditions of the capture environment. This paper addresses the problem of relighting captured textured 3D non-rigid surfaces sequences which do not represent the scene with sufficient accuracy to recover surface reflectance. We present a method for recovery of detailed normals under the assumption of Lambertian surface reflectance.

Classical shape-from-shading techniques enable estimation of the detail surface shape and normal field from images illuminated with a point light source. In a multiple camera studio environment with multiple illumination sources the irradiance at each surface point varies due to self-shadowing and is unknown without an accurate prior shape model. We combine an approximate prior shape model with shape-fromshading techniques to constrain the estimation of a normal field representing the surface detail.

A number of recent approaches have been presented to recover the observed local surface deformation of clothing from an image or video for texture and material replacement $[12][13][14][15][16][17][18][19]$. Fang and Hart [16] and Khan et al. [18] use shape-from-shading techniques on single images to estimate the normal field enabling texture substitution and material replacement. This approach has been extended to video [12][14][15][17] by tracking patterns on clothing to estimate a stable normal field over time allowing temporally coherent texture replacement. These approaches allow the appearance of non-rigid scenes to be modified but do not allow re-lighting.

Other approaches use photometric stereo techniques [6] to recover either a detailed surface description or illumination primitives which can be used for re-lighting. These techniques are usually applied to static scenes since they require the appearance to be captured for each surface point under multiple illuminations. In the case of dynamic non-rigid scenes the object deforms as the illumination changes, hence photometric stereo can only be achieved using high-speed camera $(4000 \mathrm{~Hz})$ and illumination [10] limiting the approach to short sequences. The approach presented in this paper uses standard video-rate $(25 \mathrm{~Hz})$ capture and studio illumination.

Other techniques use directional illumination and integrate multiple view observation over space and time to estimate surface reflectance [8][9]. However, this requires accurate spatial and temporal correspondence which is ambiguous in the case of materials with uniform appearance which commonly occur in clothing. Errors in correspondence lead to inaccuracies in surface normal estimates and loss of surface detail in reconstruction. The video-based approach presented in this work avoids the requirement for spatial or temporal correspondence. Surface normals are estimated from a single image frame utilising shading information to recover surface detail for uniform regions without constraints on the scene illumination.

In a studio the original, unprocessed video recordings must also be directly usable for conventional $2 \mathrm{D}$ production. and may be broadcasted immediately, hence no special lighting can be used. We make no assumptions about lighting. Our method can in principle be used in combination of arbitrary, even changing illumination in a live studio environment.

\section{Overview}

The appearance of objects is dependent on the illumination, the reflection properties and the $3 \mathrm{D}$ shape of the object. In practice however, these cannot be recovered in high enough detail to enable the physically correct computation. In a multi view studio environment the illumination is difficult to model due to the spatial characteristics of the light sources and secondary illumination reflected from the environment. Without an accurate $3 \mathrm{D}$ shape it is not possible to calculate self shadows and surface features such as creases or wrinkles. The reflective characteristic of the illuminated surface changes the appearance of the object significantly.

The general relighting problem is badly under constrained. There were several attempt to reduce the number of unknown parameters by calibration of the environment or using specialist equipment as detailed in section 2. For practical reasons we intend to use regular studio equipment only and avoid complicated light calibration. To reduce the under determined nature of the problem we perform computation over an extended patch of the surface, instead of per-pixel. The technique requires the patch to have constant Lambertian reflectivity and be large enough to cover an area with a wide range of surface normals. These assumptions are generally valid or nearly valid for textiles, however they are not valid for skin regions as discussed in section 6 .

To further reduce the ambiguity, a simplified lighting model is employed. It is assumed that each uniformly coloured surface patch is illuminated by a single unknown point light source. This is a major simplification motivated by the fact that the arbitrary illumination of a single Lambertian surface point can be modelled by a virtual point light source. However, it disregards the effect of self shadowing and occlusion resulting in a different virtual light source for every surface point.

The use of extended surface patches and a simplified illumination model reduces the ambiguity of the computation by providing multiple samples differing in only one parameter, the surface normal. The price for such simplification is the loss of accuracy and the emergence of artefacts. The proposed technique attempts to eliminate these artefacts by constraining the estimated normal map with a low detailed normal map computed from an initial surface approximation. The advantage of this approach is that it is based on the initial reconstruction and the original colour images only and does not require photometric calibration. It can also cope with arbitrary complex and changing illumination.

In this work, we introduce a system for relighting clothing of actors in virtual environments. It is based on a method to combine refined normal estimates from shape-from-shading with previous work on multiple view reconstruction to enable relighting of dynamic scenes. The approach consists of three steps. First, the object surface is segmented into regions of uniform surface reflectance using a novel multiple view temporal extension to mean-shift [20][21]. Second, the normal field within each region for each view is estimated from the image gradient. Third, estimated normals are constrained by the initial surface reconstruction to represent the fine surface detail. 
The principal contribution of this work is the combination of shape-from-shading with multiple view reconstruction to enable representation of fine surface detail for non-rigid scenes. This approach is applied to multiview capture of moving people to demonstrate re-lighting of detailed surface dynamics such as creases in clothing.

\section{Spatio-temporal segmentation}

In this section we present a segmentation algorithm to obtain consistent segmentation across multiple camera views in video sequences. Our aim is to link together image regions of uniform colour and varying intensity across multiple view sequences as illustrated in figure 1 . The method must be robust against appearance changes caused by shading and shadowing because the segmentation will serve as an input for subsequent shapefrom-shading based surface normal refinement process, as shown in figure 3, and ensures view consistency in later processing steps.

The mean-shift based region segmentation algorithms [20][22] suited for this task because they extend to higher dimensional spaces to cluster over colour, space and time. In this work we also extend the mean-shift approach to obtain consistent clustering across multiple-view video sequences. Mean-shift is a clustering algorithm based on gradient density function estimation introduced by Fukunaga and Hostler [22]. The method is suitable for vector spaces with clusters of complex shapes. Wang at al. [21] introduced video segmentation and shown that mean-shift segmentation with an anisotropic kernel performs well.

Comaniciu and Meer [20] introduced a solution to use meanshift for segmenting colour images transformed into $\mathrm{L}^{*} \mathrm{u}^{*} \mathrm{v}^{*}$ colour space extended by two spatial dimensions. Segmenting motion images frame-by-frame causes flickering due to incoherence in time. Therefore a sixth time dimension must be inserted into the vector space [21] resulting in a 6D space-timeluminance-colour vector space. Generally, an isotropic kernel is used in colour dimensions during segmentation. Since we aim to be invariant for shadows and shadings, the size of the kernel in the luminosity dimension must be relatively large.

The principal problem in extending the mean-shift approach to sequences and multiple views is computational cost which increases exponentially with the dimensionality of the feature space. The complexity of this algorithm is proportional to the fourth power of the number of observation vectors, hence increasing the number of vectors is not practical. To overcome this complexity issue we cut the video into small clips. Time coherent segmentation between clips is enforced using a sliding-window scheme, as illustrated in figure 2. Consecutive frames of the video sequence are grouped into a block and three or more blocks are combined into a window. All the frames of

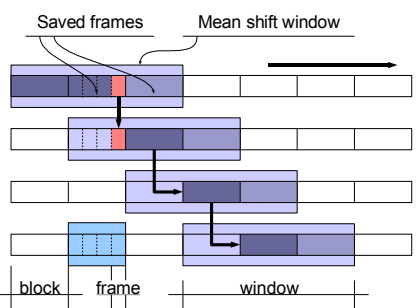

Figure 2 Outline of the sliding-window scheme

window are segmented together, but only the middle blocks are used. The first and last blocks are only necessary to eliminate edge effects arising from imposing limits on the temporal axis of the $6 \mathrm{D}$ space-time-luminance-colour space. Windows are segmented independently, however labels of image regions are synchronised between the last frames of the second last and the first blocks in two consecutive windows.

Using the sliding window technique the computational cost increases linearly with the length of the video stream and the required memory remains constant. The segmentation of a 300 frame long HD video stream is 25 times faster using the sliding window scheme.

Segmenting multiple camera views independently results in different segment indices for each view. Efficient multiple view segmentation with consistent regions labels is achieved by synchronisation between views. Correspondence between pixels observed for different views can be obtained via the 3D model created using multiple-view surface reconstruction technique. Since the segmentation is generally more accurate than the 3D model, assigning the same region indices by pair wise pixel correspondences is error-prone. Hence we assign the same index to regions in different views which are most similar.

Counting how many times segment $k$ in view $s$ belongs to segment $l$ in view $d$, we define the region correspondence matrix, $\boldsymbol{H}_{s \boldsymbol{d}}(k, l)=\eta_{k l}^{s d}$. Doing this process reversely, $\mathbf{H}_{\mathbf{d s}}$, is also necessary. If more than two camera views have to be synchronized we must search the index pairs of all camera views. All index pairs of all camera view pairs are stored in a four dimensional view correspondence matrix, $\Xi$, which is a quadratic matrix of the region correspondence matrices $\left(\mathrm{H}_{\mathrm{ij}}\right)$.

Using a perfect 3D model, and perfect segmentation all $\mathrm{H}_{\mathrm{ij}}$ matrices in $\Xi$ have at most one element which is different from zero in all rows or columns signifying perfect correspondence between regions in views. Note, that it is possible that a row or column does not contain any element which is different from zero if there is a segment which can only be seen in one view. In practice, we must find the corresponding region labels in the presence of inaccuracies in the $3 \mathrm{D}$ model and inconsistent segmentation between views.


Figure 1 View consistent segmentation of one frame of a video sequence captured from multiple camera positions. 
Since the sizes of the regions may differ significantly, the $\eta_{k l}^{i j}$ values are not directly comparable, therefore normalization is needed

$$
\bar{\eta}_{k l}^{i j}=\eta_{k l}^{i j} / \sum_{m=1}^{N_{k}^{i j}} \eta_{k m}^{i j}
$$

In the normalized view correspondence matrix $\overline{\boldsymbol{E}}$, all values are $0 \leqslant \bar{\eta}_{k l}^{i j} \leqslant 1$, where 1 means that the segment $k$ in view $i$ corresponds to segment $l$ in view $j$ perfectly.

The final region correspondences are established by selecting the most likely matches based on the normalised view correspondence matrix. Segment $k$ and $l$ in view $i$ and $j$ are merged if the corresponding $\bar{\eta}_{k l}^{i j}$ value is larger than a predefined threshold.

\section{3D model enhancement}

Our aim is to recover the fine changes of the normal vectors over the surface of the $3 \mathrm{D}$ model of a real actor created by multiple view 3D reconstruction [7]. These changes are captured in the image intensity. The relationship between intensity and surface normals at a given point is parameterised by the reflectivity of the surface point and the direction and intensity of the effective light sources - the light sources illuminating the surface point.

There are various reflection models formulated in the vision literature $[23][24][25]$. We assume that the actors in a controlled studio environment posses only diffuse reflection properties:

$$
I(\lambda, \bar{x})=\rho(\lambda, \bar{x}) \int_{L(\bar{x})} I_{d}(\lambda) \bar{n}(\bar{x}) \cdot \bar{l}_{d}
$$

Expression 1 represents the diffuse part of the reflected light, where $\rho(\lambda, \bar{x})$ and $\bar{n}(\bar{x})$ are the spectral reflectance (colour) and the surface normal at a given point $\bar{x}$ of the surface of the object, respectively. $L(\bar{x})$ is the set of effective light sources. $I_{d}(\lambda)$ and $\bar{l}_{d}$ are the spectral intensity and the direction of an elementary illuminant, respectively. In the diffuse case, any complex illumination can be substituted by a point light source for each infinitesimal surface point

$$
\overline{I_{D}}(\lambda, \bar{x})=I_{D}(\lambda, \bar{x}) \overline{l_{D}}(\bar{x})=\int_{L(x)} I_{d}(\lambda) \overline{l_{d}}
$$

where $I_{D}(\lambda, \bar{x})$ and $\overline{l_{D}}(\bar{x})$ are the intensity and direction of the virtual point light source, respectively. The observed intensity in any $3 \mathrm{D}$ surface point $\bar{x}=\left[x_{1}, x_{2}, x_{3}\right]$ is

$$
I(\lambda, \bar{x})=\rho(\lambda, \bar{x}) I_{D}(\lambda, \bar{x}) \bar{n}(\bar{x}) \cdot \bar{l}_{D}(\bar{x}) .
$$

Expression 3 means that there is a different virtual point light source for each point $\bar{x}$ due to the difference of the effective light sources $L(\bar{x})$. This difference represents cast and attached shadows. The surface normal $\bar{n}(\bar{x})$ can only be recovered if the position of this virtual point light source and the reflectivity of point $\bar{x}$ are known.

In practice, neither the reflectivity nor the virtual point light source are known. More over, the knowledge of the virtual point light source for each surface point requires the knowledge not only of the distribution of the illuminants but the 3D shape of the object in detail. Of curse, the detailed 3D shape defines the surface normals regardless of the illumination. Expression 3



Figure 3 Overview of the proposed technique

represents a coupled under defined problem. The proposed technique, presented in the next section, solves expression 3 by assuming constant reflectivity and virtual point light source over an extended area of the model surface. These assumptions yield inaccurate results in shape-from-shading, hence a subsequent correction step based on the initial surface approximation of the object is introduced. The process is illustrated in figure 3 .

\subsection{View dependent normal field recovery}

The spatio-temporal segmentation, detailed in the section 4, segments the surface of the 3D model, initially constructed using a multiple view 3D reconstruction technique, into regions of uniform surface colour. The intensity variation within these regions is due to the change of surface normals, self-shadows cast by other parts of the model - the change of the effective light source - and specularity.

The proposed method only considers the variations resulting from the change of normal vectors, does not consider textured surfaces and assumes a Lambertian surface, hence expression 3 can be reformulated

$$
I(\lambda, \bar{x})=\rho(\lambda) I_{D}(\lambda) \bar{n}(\bar{x}) \cdot \overline{l_{D}}
$$

Changes caused by the other phenomena result in errors of the normal field. These errors are corrected in a later step of the proposed method using the initial shape serving as a constraint.

The proposed normal refinement technique consists of three main steps depicted in figure 5. First, the effects of the unknown reflectivity $\rho(\lambda)$ and light intensity $I_{D}(\lambda)$ are compensated. Second, the initial normal filed $\bar{n}(\bar{x})$ is approximated, than the normal field is re-approximated using corrected illumination direction $\overline{l_{D}}$. Third, the estimated normal field is constrained by the object's initial shape. The normal refinement steps are carried out on the original camera images, yielding a view dependent normal map for each frame of each view. The view dependent normal maps are used during re-lighting to produce a view independent re-lit $3 \mathrm{D}$ model as shown in figure 6 and explained in section 5.2. 
The reflectivity and the intensity of the illumination act as a scaling factor in expression 4 . To recover the dot product of the normal vector and the direction of the illumination each region must be normalised by dividing the intensity of each pixel with the maximum intensity within the region.

$$
J(\lambda, \bar{x})=\bar{n}(\bar{x}) \cdot \Gamma_{D} \approx \frac{I(\lambda, \bar{x})}{\max _{x^{\prime} \in R_{i}} I\left(\lambda, \bar{x}^{\prime}\right)},
$$

where $\bar{x} \in R_{i}$ and $R_{i}$ represents the $i^{\text {th }}$ region. This assumes that there is a point in every region where the normal vector points towards the effective light source.

Based on the normalised intensities the surface normal can be estimated by the technique of Fang and Hart [16].

$$
\begin{gathered}
G(\bar{x})=\nabla I(\bar{x})-\left(\nabla I(\bar{x}) \cdot \bar{l}_{D}\right) \bar{l}_{D} \\
n(\bar{x})=J(\bar{x}) \bar{l}_{D}+\sqrt{1-J(\bar{x})^{2}} \frac{G(\bar{x})}{\|G(\bar{x})\|}
\end{gathered}
$$

where $\nabla I(\bar{x})=(\partial I / \partial u, \partial I / \partial v, 0)$ is the image gradient in any image of the actor at surface point $\bar{x}$ and $(\mathrm{u}, \mathrm{v})$ are the corresponding pixel co-ordinates in the image. Based on expression 7, the normal vector at any points of the model surface can be approximated only if the direction of the illumination is known. The proposed technique estimates the normal vector in two consecutive iterations, depicted in figure 5. During the first iteration, an initial illumination direction is placed at the camera centre and used to calculate the image gradient. Placing the illumination at the camera centre ensures that all visible surface points are illuminated. The normal field computed using the initial light source direction differs from the correct normal filed. This difference is approximated by calculating the rotation matrix bringing the estimated normal field and the normal field of the 3D model into the best alignment in a least square sense. This problem is analogous to the pose estimation problem.

$$
\operatorname{Rot}=\operatorname{argmin}\left(\sum_{\bar{x} \in R_{i}} w(\bar{x})\left\|n_{\text {visual hull }}(\bar{x})-\operatorname{Rot} n(\bar{x})\right\|^{2}\right)
$$

where

$$
w(\bar{x})=\left\{\begin{array}{lll}
1 & \text { if } & \|\nabla I(\bar{x})\|<\nabla_{\max }\left(R_{i}\right) / 2 \\
0 & \text { if } & \|\nabla I(\bar{x})\| \geq \nabla_{\max }\left(R_{i}\right) / 2
\end{array}\right.
$$

and

$$
\nabla_{\max }\left(R_{i}\right)=\max _{\bar{x} \in R_{i}}\|\nabla I(\bar{x})\|
$$

where $\nabla_{\text {max }}\left(R_{i}\right)$ is the maximum image gradient in the $i^{\text {th }}$ region. The weighting factor is applied to eliminate points with large gradient value from the minimisation process. These points may offset the estimated rotation since normal field

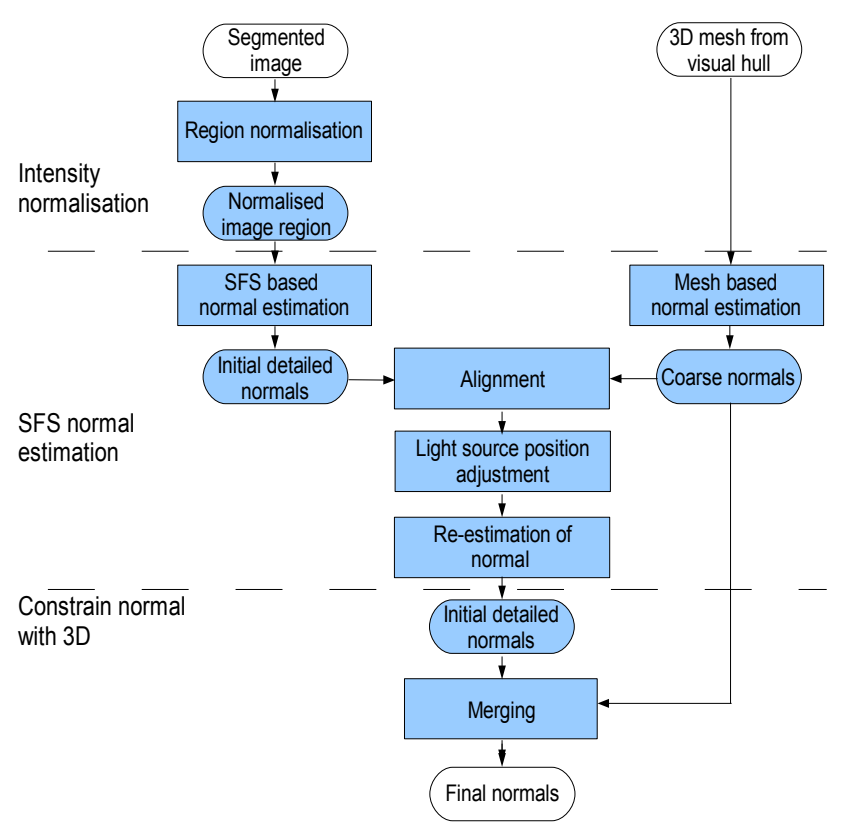

Figure 5 Outline of view dependent normal recovery process

based the surface reconstruction is smooth and does not represent rapid changes.

In the second iteration, the initial illumination direction is modified by applying the rotation between the two normal fields. The final normal fields is calculated by applying expression 7 with the modified illumination direction.

The normal field acquired as the result of the second iteration step of the proposed method represents the fine structures of the surface, however it is inaccurate as shown in figure 4 . The inaccuracy is due to modelling the illumination by a single point light source. Though for diffuse surfaces, the illumination can be represented by one point light source, this is different for every point of the surface. The last step of the normal field recovery process is to eliminate inaccuracies by merging the low detail normal field of the reconstructed surface with the high detail normal field of the proposed method.

The likelihood of the presence of surface details are higher at points where the image gradient is larger, hence the weighted average of the two normal fields is calculated.

$$
n_{\text {final }}(\bar{x})=(1-c) n_{\text {visual hull }}(\bar{x})+c n(\bar{x})
$$

where the weighting factor is
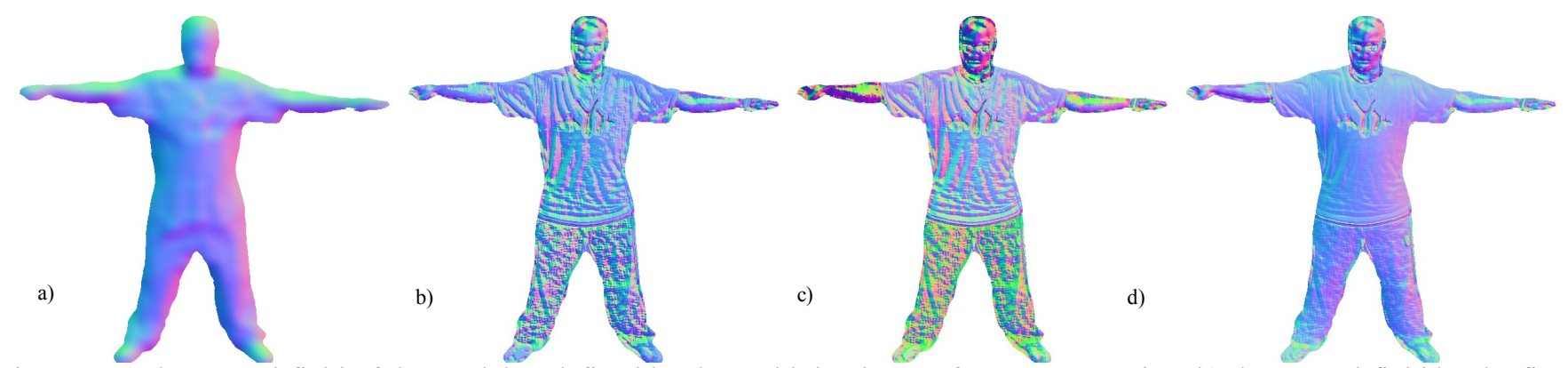

Figure 4. a) the normal field of the model as defined by the multiple-view surface reconstruction; b) the normal field by the first pass; c) the normal field after the second pass of the proposed recovery technique; d) the final normal field recovered. 


$$
c=\sqrt{\frac{\|\nabla I(\bar{x})\|}{\nabla_{\max }\left(R_{i}\right)}}
$$

and $n_{\text {final }}$ is the final result of the normal vector recovery process representing the normals for each view independently.

\subsection{Viewpoint independent re-lighting}

After recovering the normal vectors of the model of an actor, the model can be put into a new virtual environment and the effect of the lighting environment of the virtual world can be calculated.

A viewpoint dependent re-lighting is performed from the viewpoints of the original cameras resulting in re-lit images of the model. First, the view dependent normal images are mapped to the 3D model using projective texture mapping. Second, the 3D model is rendered in the virtual environment from the viewpoint of the original cameras using the assigned normals and the 3D shape. Self shadows are computed according to the initial shape reconstruction whilst shading is calculated according to the recovered normals. The colour of each surface region is assigned manually according to the segmentation of the model and follows a Lambertian reflection model.

The re-lit images are combined into a texture map covering the entire surface of the 3D model. This enables showing the re-lit 3D model from arbitrary view points as illustrated in figure 6 . In our experience the re-lit images are consistent, therefore no additional steps are needed to merge them into one texture map.

\section{Evaluation of recovered surface detail for re- lighting}

Our aim is to recover surface features to enable re-lighting of clothing using standard studio equipment only. It is assumed that the materials used consist of large single colour Lambertian patches. To evaluate the performance of the proposed technique, we captured actors performing from 9 different viewpoints in a blue box studio illuminated by a number of stage lights. The illumination is neither calibrated nor recorded.

The actors were performing rapid movements, hence their clothing was changing continuously. The $3 \mathrm{D}$ reconstruction was carried out using the multiple view reconstruction technique presented in [7]. The surface normals were estimated by the proposed method on every frame for each view independently. To visualise the results, the 3D models were relit under artificial illumination using a simple point based Lambertian rendering model. Out aim was not to produce realistic renderings by using advanced rendering techniques but to demonstrate the increase of the surface details. We believe that using production quality rendering engines, a more realistic appearance could be achieve.

Figure 7 (middle row) shows four frames of a video sequence and these four frames re-lit under two different illumination conditions (top/bottom rows). The middle row shows renderings of the textured initial smooth 3D surface models. The 3D model lacks details of cloth creases, as illustrated in figure 4. Fine surface details are only represented in the coloured texture map. Hence, changing the illumination does not change the appearance of surface.

The top and bottom rows in figure 7 illustrate the effect of the changing environment. The appearance of folds on the T-shirt and the shadows on the body change according to the change of the illumination direction. These 3D models are enhanced using the proposed technique therefore more detailed normal vectors are attached to each surface point. The estimated normal field is represented as a normal map on the initial smooth 3D surface. Accompanying videos illustrate how the creases in the clothing are consistently relit for sequences of the person moving.

Though the appearance of the model frames are different in the top and bottom rows, the models are the same, respectively. The difference is the result of the varying direction of the illumination.

The segmentation algorithm does not reliably segment the face and hair which have similar colour into different regions. Consequently the assumption of uniform reflectance is violated in the face region resulting in erroneous normal estimates. The resulting normal map on the face improves the detail over the smooth model as illustrated in figure 8. However, re-lighting the face results in visual artefacts due to incorrect normals, lack of colour details - e.g. hair, eyes, lips have the same colour and simplified reflection model used in re-lighting. Realistic rendering of skin regions require advanced graphics techniques which is the subject of our future research.

Figure 8 shows another illustration of the same technique. The actor in this figure is well illuminated making possible a full recovery of shirt, trouser and skin regions. The light source applied in c) in figure 8 is a complex area light, creating smooth shadows both on the face and the clothing. The unrealistic look of the face region is noticeable in these images similarly to figure 7 . This appearance can be radically altered by applying different illumination. The same refined model is pictured in d) in figure 8 lit by a point illuminant. The direction of the illumination is closer to the view direction than in the images in c) therefore the effects of shadowing is less prominent.



Figure 6 different viewpoints of the same relit actor illustrating that the proposed technique is viewpoint independent 


\section{Conclusion and future work}

This paper has introduced an approached to relighting reconstructed 3D non-rigid surfaces with realistic reproduction of details such as creases in clothing. The approach is based on the combination of an initial 3D surface approximation with details represented using normals estimated from shading. A novel approach has been introduced to combine the noisy normal estimates with a smooth initial surface approximation assuming segmented surface regions of uniform colour. Normals mapped onto the 3D surface from multiple views reproduce the dynamics of surface detail during movement allowing relighting and viewpoint change. This overcomes limitations of previous direct re-lighting of reconstructed models which fail to reproduce dynamics of surface detail.

Future research is required to improve the re-lighting of detailed surface regions such as the face and extend the approach to non-Lambertian surfaces.

\section{References}

[1] T. Kanade, P. Rander and P.J Narayanan. Virtualized reality: constructing virtual worlds from real scenes. IEEE Multimedia, 4(1):34-47, 1997.

[2] W. Matusik, C. Buehler, R. Raskar, L. McMillan and S. Gortler. Image-Based Visual Hulls. Proceedings of Siggraph, $369-374,2000$

[3] C. Theobalt, M. Magnor, P. Schueler, H.P. Seidel. Combining 2D Feature Tracking and Volume Reconstruction for Online Video-Based Human Motion Capture. Proceedings of 7 th International Fall Workshop on Vision, Modeling and Visualization, 96-103, 2002

[4] S. Vedula, S. Baker, S. Seitz, and T. Kanade. Shape and Motion Carving in 6D. Proc. Computer Vision and Pattern Recognition, 592-598, 2000

[5] L.C. Zitnick, S.B. Kang, M. Uyttendaele, S.A.J. Winder and R. Szeliski. High-quality Video View Interpolation Using a Layered Representation. Proceedings of ACM SIGGRAPH, 600-608, 2004

[6] G. Vogiatzis, C. Hernández and R. Cipolla. Reconstruction in the Round Using Photometric Normals and Silhouettes. IEEE Conference on Computer Vision and Pattern Recognition, 1847-1854, 2006

[7] J.Starck and A.Hilton. Surface Capture for Performance based Animation. IEEE Computer Graphics and Applications, 2131,2007

[8] C Theobalt and N. Ahmed. The Relightable Free-Viewpoint Video Project. Technical Report: (http://www.mpiinf.mpg.de/ theobalt/RFVV/index.html), ,

[9] C.Theobalt, N.Ahmed, M.Magnor and H.P.Seidel. . IEEE Trans. Visualization and Computer Graphics, , 2007

[10] P. Einarsson, CF. Chabert, A. Jones, WC. Ma, B. Lamond, T. Hawkins, M. Bolas, S. Sylwan, and P. Debevec. Relighting Human Locomotion with Flowed Reflectance Fields. 17th Eurographics Symposium on Rendering, 183-194, 2006

[11] A. Hertzmann, S.M. Seitz. Example-based photometric stereo: shape reconstruction with general, varying BRDFs. IEEE Transactions on Pattern Recognition and Machine Intelligence, 27(8):1254 - 1264, 2005.

[12] A. Lobay and D.A. Forsyth. Recovering shape and irradiance maps from rich dense texton fields. Conference on Computer Vision and Pattern Recognition, 400-406, 2004

[13] S. Zelinka, H. Fang, M. Garland, and J. Hart.. Interactive Material Replacement in Photographs. Graphics Interface, 227-232, 2005.
[14] V. Scholz and M. Magnor. Texture Replacement of Garments in Monocular Video Sequences. Rendering Techniques 2006 (Proc. of the 17th Eurographics Symposium on Rendering EGSR 2006), 305-312, 2006

[15] R.M. White and D. Forsyth. Retexturing Single Views Using Texture and Shading. Electrical Engineering and Computer SciencesUniversity of California at Berkeley Technical Report No. UCB/EECS-2005-4, 2005.

[16] H. Fang and J. Hart. Textureshop: Texture Synthesis as a Photograph Editing Tool. ACM Trans. Graph, 23():354-359, 2004.

[17] H. Fang and J.C. Hart. Roto Texture: Automated Tools for Texturing Raw Video. IEEE Transactions on Visualisation and Computer Graphics, 12(6):1580-1589, 2006.

[18] E.A. Khan, E. Reinhard, R. Fleming and H. Buelthoff. Imagebased Material Editing. ACM Transactions on Graphics (Proceedings of Siggraph), 25(3):654-663, 2006.

[19] M. Salzmann, S. Ilic and P. Fua. Physically Valid Shape Parameterization for Monocular 3-D Deformable Surface Tracking. British Machine Vision Conference, , 2005

[20] D. Comaniciu and P. Meer. Mean Shift: A Robust Approach Toward Feature Space Analysis. IEEE Transactions on Pattern Analysis and Machine Intelligence, 24(5):603-619, 2002.

[21] J. Wang, Y. Xu, H. Shum and M. Cohen. Video Tooning. Proceedings of ACM SIGGRAPH, 574-583, 2003

[22] K. Fukunaga and L. Hostetler. The estimation of a gradient density function, with applications in pattern recognition. IEEE Trans. Information Theory, 21():32-40, 1975.

[23] S.K. Nayar, K. Ikeuchi and T. Kanade. Surface Reflection: Physical and Geometrical Perspective. IEEE T. Pattern Anal., 13(7):611-634, 1991.

[24] X.D. He, K.E. Torrace, F.X. Sillion and D.P. Greenberg. A Comprehensive Physical Model for Light Reflection. Computer Graphics, 25():175-186, 1991.

[25] Y. Yu and J. Malik. Recovering Photometric Properties Of Architectural Scenes From Photographs. Proc. SIGGRAPH 98, 207-217, 1998. 


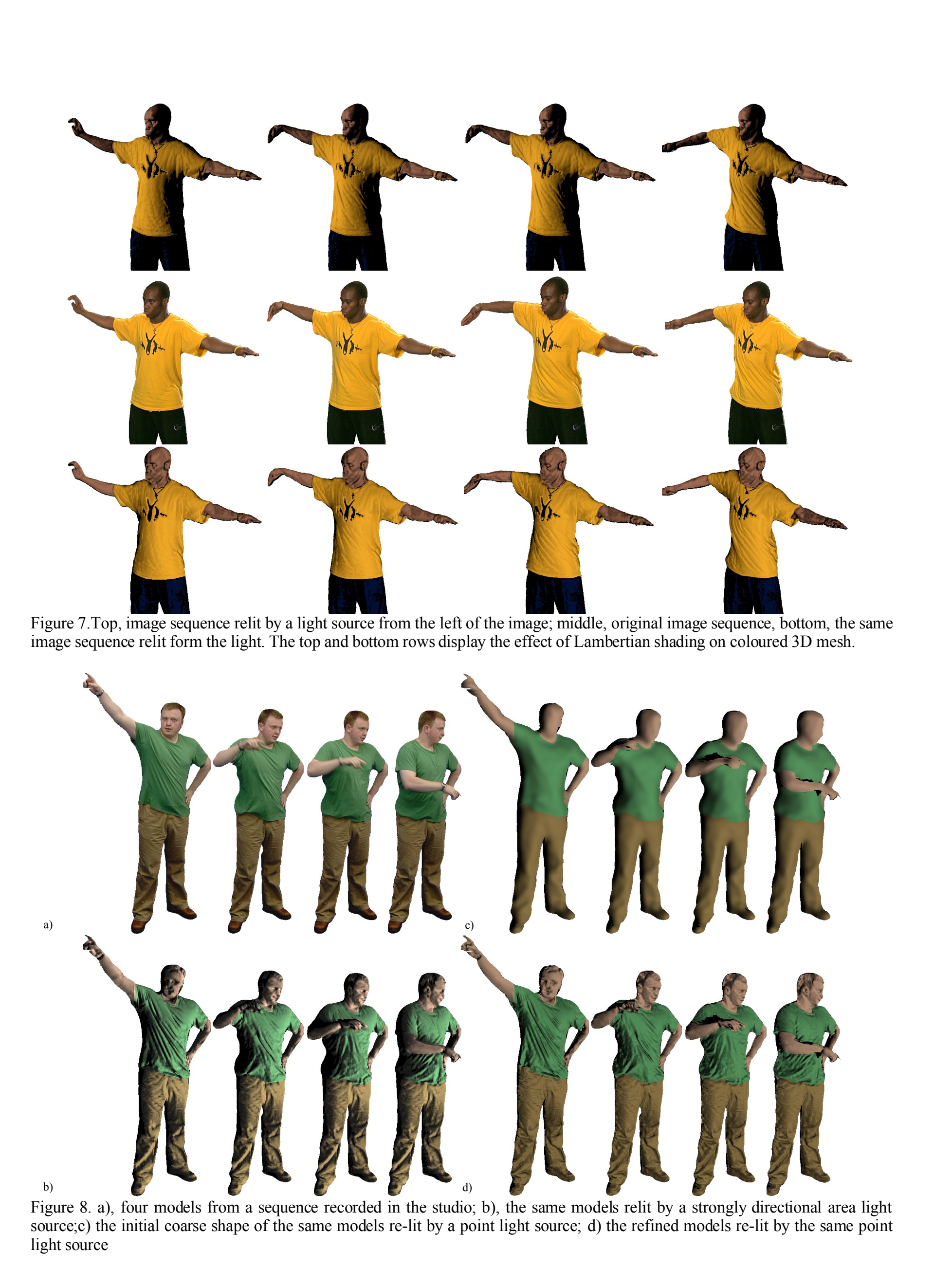

